\title{
Neck Lymph Node Boundary Sublevel VA
}

National Cancer Institute

\section{Source}

National Cancer Institute. Neck Lymph Node Boundary Sublevel VA. NCI Thesaurus.

Code C132501.

The following anatomical structures define the neck lymph node boundary for sublevel VA: Superior: apex of the convergence of the sternocleidomastoid and trapezius muscles; inferior: horizontal plane defined by the lower border of the cricoid cartilage; anterior (medial): posterior border of the sternocleidomastoid muscle or sensory branches of the cervical plexus; posterior (lateral): anterior border of the trapezius muscle. (AJCC 8th ed.) 